\title{
Bloch-Zener oscillations in graphene and topological insulators
}

\author{
Viktor Krueckl and Klaus Richter \\ Institut für Theoretische Physik, Universität Regensburg, D-93040 Regensburg, Germany \\ (Received 27 September 2011; revised manuscript received 9 March 2012; published 22 March 2012)
}

\begin{abstract}
We show that superlattices based on zero-gap semiconductors such as graphene and mercury telluride exhibit characteristic Bloch-Zener oscillations that emerge from the coherent superposition of Bloch oscillations and multiple Zener tunneling between the electron and hole branch. We demonstrate this mechanism by means of wave-packet dynamics in various spatially periodically modulated nanoribbons subject to an external bias field. The associated Bloch frequencies exhibit a peculiar periodic bias dependence, which we explain within a two-band model. Supported by extensive numerical transport calculations, we show that this effect gives rise to distinct current oscillations observable in the $I-V$ characteristics of graphene and mercury telluride superlattices.
\end{abstract}

DOI: 10.1103/PhysRevB.85.115433

PACS number(s): 72.80.Vp, 73.21.Cd, 85.35.Ds, 85.75.Mm

\section{INTRODUCTION}

Bloch oscillations, the periodic motion of particles in a superlattice subject to a constant external field, represent a fundamental phenomenon in transport through periodic potentials. Predicted in the early days of quantum mechanics, ${ }^{1,2}$ Bloch oscillations have been observed in various fields of physics, ranging from earlier experiments in semiconductor superlattices $^{3-5}$ via cold atoms in optical lattices ${ }^{6,7}$ to classical optical $^{8,9}$ and acoustic ${ }^{10}$ waves. While many aspects of conventional Bloch oscillations can be explained by a single band description, particularly interesting effects arise in the case of two coupled minibands ${ }^{11}$ energetically separated from further bands. Then partial Zener tunneling at avoided crossings of the two minibands can lead to a coherent superposition of Bloch oscillations, ${ }^{12,13}$ i.e., to a splitting, followed by a subsequent recombination of a Bloch oscillating wave packet. This gives rise to a variety of Rabi-type interference phenomena, in particular double-periodic motions coined Bloch-Zener (BZ) oscillations. ${ }^{14-16}$ Signatures of this effect have already been detected in the THz emission of AlGaAs superlattices, ${ }^{17}$ and even the population dynamics has been measured recently for light ${ }^{18}$ and atomic matter waves ${ }^{19}$ in specifically tailored binary lattices.

However, materials with a linear Dirac spectrum ${ }^{20}$ naturally serve the effect, since only a small gap is opened by a spatially periodic modulation allowing for Zener tunneling between electron and hole states. Such materials are now at hand with the discovery of graphene $e^{21,22}$ and the advent of topological insulators ${ }^{23-26}$ first realized in two-dimensional mercury telluride (HgTe) heterostructures. ${ }^{27,28}$ Interesting phenomena for graphene-based periodic superstructures have already been theoretically predicted, such as the formation of extra Dirac cones ${ }^{29-31}$ and the appearance of a negative differential conductance. ${ }^{32}$ Furthermore, recent experiments have realized graphene superlattices with periodicities down to a few nanometers. ${ }^{33}$

This raises the question of the existence of peculiarities of Bloch oscillations in graphene and topological insulator superlattices, which we address in this paper. We are not aware of any work showing unconventional features in graphenebased Bloch oscillations. Until now, only the semiclassical approach has been adapted to a linear dispersion ${ }^{34}$ and, without reference to Bloch oscillations, numerical evidence for a negative differential conductance was reported. ${ }^{32}$ We show that besides conventional Bloch oscillations, multiple Zener tunneling between the coupled electron and hole branches leads to distinct $\mathrm{BZ}$ oscillations that appear to be naturally present in superlattices made of systems with Dirac-like dispersion.

This paper is structured as follows: In Sec. II, we show the influence of BZ oscillations on the wave-packet motion in a graphene nanoribbon and the influence on the frequency spectrum. Subsequently, we introduce in Sec. III a two-band model to explain the effect in the frequency spectrum and the influence of BZ oscillations on the electron-hole polarization. In Sec. IV, we show that the occurrence of BZ oscillations can be seen as distinct features in the current through graphene nanoribbons. In Sec. V, we present results that feature the special frequency pattern of BZ oscillations, as well as their signatures in transport, in nanoribbons made of the topological insulator mercury telluride.

\section{WAVE-PACKET MOTION IN GRAPHENE SUPERLATTICES}

An insight into the dynamics of $\mathrm{BZ}$ oscillations can be gained by the time evolution of a wave packet on a graphene nanoribbon in the presence of a periodic mass potential $M(x)$ and a linear electrostatic drift potential $V(x)$, as sketched in Fig. 1(a). To this end, we model the electronic structure of graphene by a conventional tight-binding Hamiltonian, ${ }^{35}$

$$
H_{\mathrm{tb}}=\sum_{\langle i j\rangle, \beta} t c_{i,-\beta}^{\dagger} c_{j, \beta}+V c_{i, \beta}^{\dagger} c_{i, \beta}+M \beta c_{i, \beta}^{\dagger} c_{i, \beta},
$$

where $\langle i j\rangle$ denotes neighboring unit cells and $\beta= \pm 1$ denotes the sublattice degree of freedom. The initial wave packet is created by diagonalizing the periodic Hamiltonian $H(k)$ of one unit cell of the infinite ribbon. By means of the transversal eigenfunctions $\chi_{n}(y, k)$, we create an initial electronlike wave packet

$$
\psi_{n}(x, y)=\int_{-\infty}^{\infty} \chi_{n}(y, k) e^{\mathrm{i} k x} e^{-\frac{1}{2} k^{2} \delta^{2}} d k,
$$

with a Gaussian broadening $\delta$. Since the armchair boundary mixes the two graphene valleys, the wave function comprises several nodes in the lateral direction. The time evolution is 


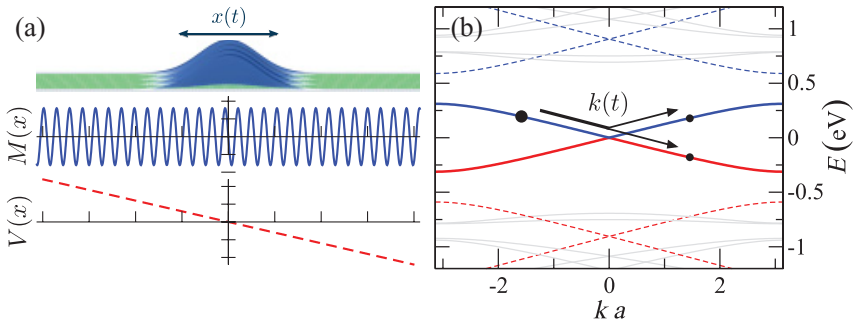

FIG. 1. (Color online) Exemplary setup for Bloch-Zener oscillations in a graphene nanoribbon. (a) Sketch of a Gaussian wave packet in the presence of a periodic mass potential $M(x)=M_{0} \sin (2 \pi x / a)$ and an electrostatic drift potential $V(x)=-e E_{D} x$. (b) Band structure of the superlattice with small avoided crossing at $k=0$ (nanoribbon width $W=10 a_{0}, a=10 \sqrt{3} a_{0}, M_{0}=0.1 t$ ). Thick and dashed lines show the first and second Bloch band from the metallic armchair mode. The gray lines represent higher transversal modes.

calculated by an expansion of the time-evolution operator in Chebychev polynomials. ${ }^{36}$ In the presence of a periodic mass potential

$$
M(x)=M_{0} \sin (2 \pi x / a),
$$

where $M_{0}$ is the strength of the periodic mass and $a$ is the periodicity length, the band structure of the superlattice exhibits a small anticrossing at $k=0$ and a large band gap between the first and the higher Bloch bands, as shown in Fig. 1(b). In the presence of a linear drift potential

$$
V(x)=-e E_{D} x,
$$

with $E_{D}$ as the strength of the drift field, the wave packet starts to accelerate. Because of its extent in the longitudinal direction, the wave packet is localized in momentum space with a distinct average momentum $k(t)$ in the $x$ direction. Given the periodicity of the band structure, a sawtooth behavior of $k(t)$ is obtained known as Bloch oscillations.

However, the dynamics in a graphene nanoribbon shows additional features due to the strong coupling between the electron and hole states. Therefore, we study a typical trajectory

$$
x(t)=\langle\psi(t)|\hat{x}| \psi(t)\rangle
$$

of the center of mass (COM), as shown in Fig. 2(a). Initially, the wave packet is chosen to be electronlike, and a snapshot of the probability distribution is shown in Fig. 2(b0). During the first Bloch cycle, the probability distribution is predominantly to the right of the initial position [see Fig. 2(b1)]. This region features a negative electrostatic potential, and accordingly the part of the wave packet with electron character performs Bloch oscillations in this region. In Fig. 2(b2), the electron and hole parts meet again in momentum space and, as a consequence, tunneling from the electron to the hole branch is possible, as sketched by the bullets in Fig. 1(b). As a result, the holelike part of the wave packet increases and, in subsequent time steps, the COM trajectory reaches negative values. The corresponding snapshot at the turning point of the the wave function in Fig. 2(b3) shows a big holelike state on the left side and a smaller electronlike state on the right side. After the next tunneling, the probability distribution between the electron and hole states is almost equal, thus the COM motion is strongly

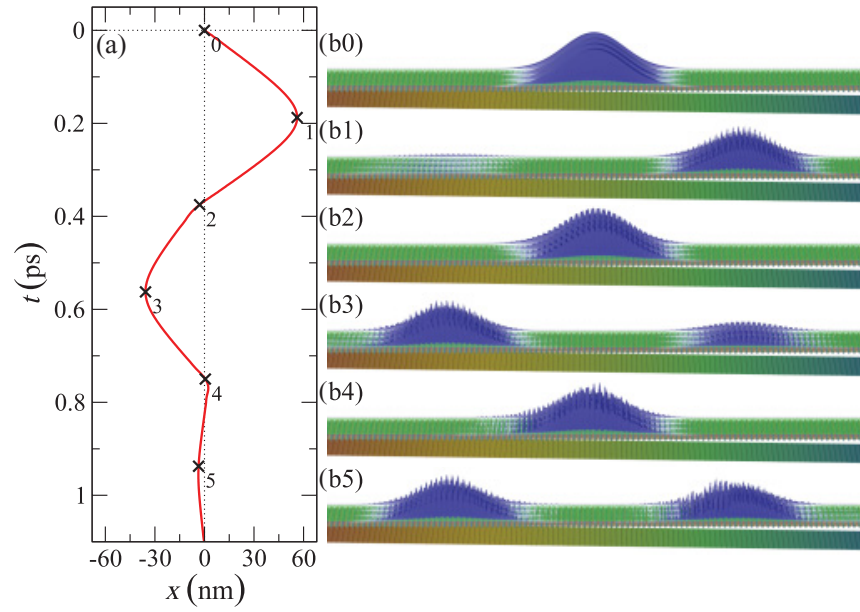

FIG. 2. (Color online) Snapshots of a wave packet in the course of Bloch-Zener oscillations. (a) Center-of-mass motion of a wave packet on a graphene nanoribbon $\left(W=10 a_{0}, a=10 \sqrt{3} a_{0}, M_{0}=0.1 t\right)$. (b) Snapshots of the probability distribution of the wave packet for the corresponding times marked with crosses in panel (a). Please note the video of the dynamics in the online version of the Supplemental Material (Ref. 37).

suppressed. Because of the periodic mass potential, the gaps between the first Bloch band and higher bands is bigger than the gap between the electron and hole states, as shown in Fig. 1(b). As a result, the tunneling into higher bands is very unlikely and there is no damping of the oscillations due to leakage into higher bands.

To study the dynamics of the tunneling between the electron and the hole branch in more detail, we perform a frequency analysis of the COM motion for different fields $E_{D}$. The Fourier amplitudes of the dominant frequency contributions are visualized by dark colors in Fig. 3. Besides the conventional Bloch frequency marked by a white dashed line, the resulting spectrum shows a pronounced interweaving pattern around half of this frequency (black dashed line). A stronger periodic potential, and thereby an increased gap between the electron and hole branch, leads to a rhombic structure, as shown in Fig. 3(b). These periodic features in the frequency spectrum arise from the interplay between Bloch oscillations and the
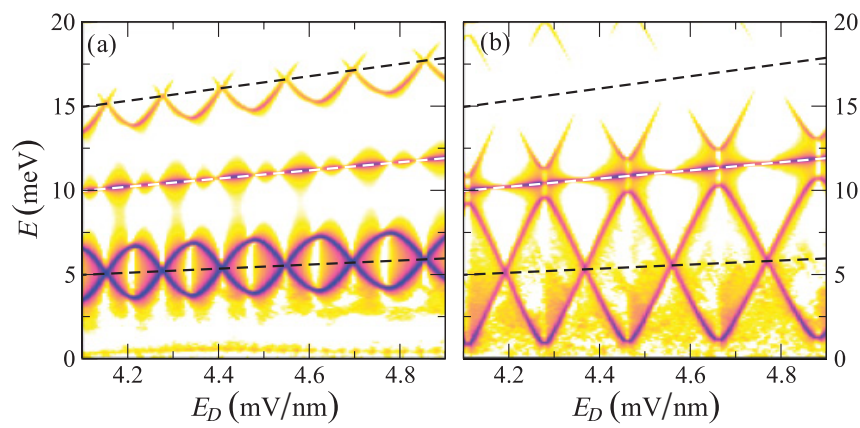

FIG. 3. (Color online) Frequency spectra $E=\hbar \omega$ from the center-of-mass motion of a wave packet for varying drift field $E_{D}$ for (a) moderate $\left(M_{0}=0.1 t\right)$ and (b) stronger $\left(M_{0}=0.2 t\right)$ periodic potential. Dark colors represent strong intensities. The dashed lines correspond to $\{1 / 2,1,3 / 2\}$ times the conventional Bloch frequency. 

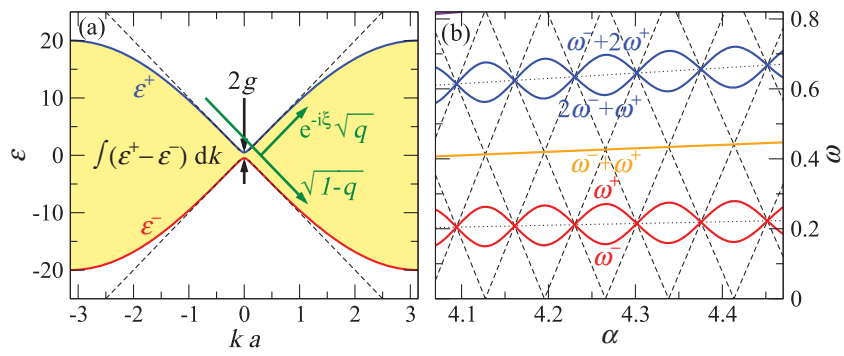

FIG. 4. (Color online) (a) Band structure of the Dirac model Hamiltonian (6) for $v=1, \hbar=1, a=1 / 10$, and $g=1 / 2$. The shaded (yellow) area denotes the integral (9). (b) Frequency spectrum of the Bloch oscillations for different drift accelerations $\alpha=e E_{D} / \hbar$. Solid lines show the frequencies $n \omega^{+}+m \omega^{-}$given by Eq. (16); dotted (dashed) lines show the strong (weak) tunneling limit.

splitting of the wave packet into the electron and hole branches at $k(t)=0$ [see Fig. 1(a)]. The persistent sequence of tunneling events between the two branches and the subsequent interference leads to a new set of frequencies, which can be understood by means of the following model.

\section{ANALYTICAL MODEL FOR BZ OSCILLATIONS}

In the following, we quantitatively explain these characteristic BZ features using a periodically modulated onedimensional Dirac model Hamiltonian,

$$
H(t)=\frac{2 \hbar v}{a} \sin \left(\frac{a k(t)}{2}\right) \sigma_{z}+g \sigma_{x} .
$$

Here, $a$ is the period, $v$ is the Fermi velocity, and $g$ is the energy gap between the electron and the hole states. The resulting band structure is given by

$$
\epsilon^{ \pm}(t)= \pm \sqrt{g^{2}+2(\hbar v / a)^{2}[1-\cos (a k)]}
$$

as shown in Fig. 4(a). A comparison with the full tight-binding calculation of the graphene nanoribbon in Fig. 1(b) shows a very good correspondence. An external electric drift field $E_{D}$ enters the equations of motion for the quasimomentum $k(t)$ as $\hbar \partial_{t} k(t)=e E_{D}$, leading to a time evolution of $k(t)=\alpha t$ linear in $t$ where $\alpha=e E_{D} / \hbar$. Conventional Bloch oscillations with frequency $\omega_{B}=\alpha a$ arise from the periodicity of $k(t)$ in momentum space in the interval $\left[-\frac{\pi}{a}, \frac{\pi}{a}\right]$. The phase $\phi$ between the two branches accumulated during one oscillation is given by a free propagation and thus

$$
\phi=\frac{\mathcal{A}}{e E_{D}} \approx \frac{16 v}{a^{2} \alpha},
$$

in which

$$
\mathcal{A}=\int_{-\pi / a}^{\pi / a}\left(\epsilon^{+}-\epsilon^{-}\right) d k
$$

is the area in momentum space, as depicted in Fig. 4(a). This free propagation can be expressed by the matrix

$$
U_{0}=\left(\begin{array}{cc}
e^{i \phi / 2} & 0 \\
0 & e^{-i \phi / 2}
\end{array}\right) \text {. }
$$

In addition to conventional Bloch oscillations on either branch, there is a strong periodic tunneling between the electron and the hole states close to the anticrossing at $k=0$. There, the
Hamiltonian (6) can be linearized [dashed lines in Fig. 4(a)], leading to a typical Landau-Zener tunneling problem: ${ }^{38-40}$

$$
H_{\mathrm{LZ}}=\left(\begin{array}{cc}
\hbar v \alpha t & g \\
g & -\hbar v \alpha t
\end{array}\right) .
$$

The scattering between the different branches is described by

$$
S_{0}=\left(\begin{array}{cc}
e^{-i \xi} \sqrt{q} & \sqrt{1-q} \\
\sqrt{1-q} & -e^{i \xi} \sqrt{q}
\end{array}\right)
$$

with the tunneling rate $q=1-e^{-2 \pi \delta}, \delta=\frac{g^{2}}{2 \hbar^{2} v \alpha}$, and $\xi=\frac{\pi}{4}+$ $\arg (1-i \delta)+\delta(\log \delta-1)$ is an additional tunneling phase. From this, we can deduce the scattering matrix

$$
S=\left(\begin{array}{cc}
e^{i(\phi / 2-\xi)} \sqrt{q} & \sqrt{1-q} \\
\sqrt{1-q} & -e^{i(\xi-\phi / 2)} \sqrt{q}
\end{array}\right),
$$

which describes the time evolution of the electron and hole branch for one Bloch cycle. Using this matrix, we derive scattering eigenstates

$$
\chi^{ \pm}=\frac{1}{\sqrt{\mathcal{N}}}\left(\begin{array}{c}
\sqrt{q} \cos (\phi / 2-\xi) \pm \sqrt{1-q \sin ^{2}(\phi / 2-\xi)} \\
\sqrt{1-q} e^{i \phi / 2}
\end{array}\right)
$$

with the corresponding eigenvalues $e^{i \beta^{ \pm}}$, where

$$
\beta^{ \pm}=\arccos \left[ \pm \sqrt{1-q \sin ^{2}(\phi / 2-\xi)}\right] .
$$

The phases $\beta^{ \pm}$of the scattering eigenstates depend periodically on the phase difference $\phi$ between the electron and hole branch. This periodicity leads to two new Bloch frequencies

$$
\omega^{ \pm}=\frac{\alpha a}{\pi} \arccos [ \pm \sqrt{q} \sin (\phi / 2-\xi)] .
$$

Unlike conventional Bloch oscillations, these frequencies do not simply depend linearly on the drift strength $\alpha$, but show a rapid interweaving pattern strongly changing with $\alpha$, as shown in Fig. 4(b), owing to coherences from combined dynamics on the hole and electron branch. The limiting cases can be understood as follows. For strong coupling, the tunneling rate $q \rightarrow 0$ leads to a frequency $\omega^{ \pm} \rightarrow \omega_{B} / 2$ [dotted line in Fig. 4(b)], since for every Bloch cycle the states tunnel completely between the two branches in momentum space and hence the complete cycle in position space is twice as long. In the opposite, weak coupling limit, $\omega^{ \pm} \rightarrow a \alpha[1 / 2 \pm(\phi / 2-$ $\xi) / \pi] \bmod 1$, leading to a rhombic frequency pattern, shown as dashed lines in Fig. 4(b). For intermediate tunneling rates, the frequencies show a smooth transition between these limiting cases and are in very good agreement with the numerically calculated spectra of Figs. 3(a) and 3(b).

Furthermore, the scattering eigenstates show a strong polarization dependence (electron- or hole-type character) on the phase $\phi$, given by Eq. (8). If the one-dimensional model Hamiltonian (6) is considered for $g^{2} \gg 2 \hbar^{2} v \alpha$, then the tunneling rate $q \rightarrow 1$, which results in strongly electronor hole-polarized states $\chi^{ \pm}$for almost all values of $\phi$. The absolute value of the spinor entries is always very close to one or zero, as shown in Fig. 5(a). Nevertheless, the polarization breaks down whenever the difference between the phase of the electron and hole branch is

$$
\phi=2(n \pi+\xi)+\pi,
$$




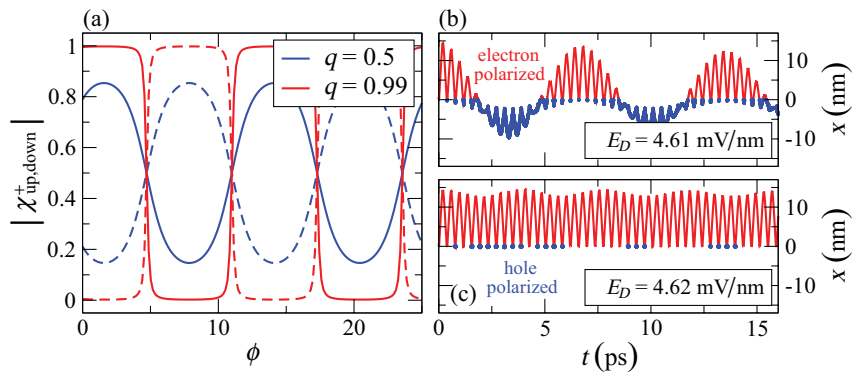

FIG. 5. (Color online) (a) Polarization dependence on the phase difference between the electron and hole branch of the scattering eigenstate $\chi^{+}$(solid line shows the upper spinor entry; dashed line shows the lower spinor entry). (b),(c) Center-of-mass motion of an initially electron-polarized wave packet on a graphene nanoribbon superlattice $M(x)=M_{0}+V(x)$ for different drift fields $E_{D}$ $\left(M_{0}=50 \mathrm{meV}, V(x)=300 \mathrm{meV} \sin (2 \pi x / a), a=10 \sqrt{3} a_{0}\right)$. Blue dots depict regions with a negative amplitude, corresponding to a wave packet with strong hole character.

where $n \in \mathbb{N}$. This alternating weight of the spinor between the electron- and hole-type states for different drift fields $E_{D}$ can be also deduced from the COM motion of wave packets with fixed initial polarization. If the drift field is adjusted such that the phase condition (17) is approximately satisfied, then the COM motion of the initially electronlike configuration exhibits oscillations ranging from -15 to $15 \mathrm{~nm}$ for $E_{D}=4.61 \mathrm{mV} / \mathrm{nm}$, as shown in Fig. 5(b). Since conventional Bloch oscillations in a single band are restricted to positive or negative values, the trajectories imply strong tunneling between the electron and hole states. For values of $E_{D}$ where condition (17) is not fulfilled, e.g., $E_{D}=$ $4.62 \mathrm{mV} / \mathrm{nm}$ in Fig. 5(c), the trajectories of the different polarizations do not significantly cross the origin, thus they preserve their electron-hole character. As a consequence, if charge transport through a system comprises a transition from electron to hole states, the current should strongly depend on the $\mathrm{BZ}$ oscillations within the superstructure.

\section{TRANSPORT IN GRAPHENE-BASED SUPERLATTICES}

In the following, we consider charge transport through graphene-nanoribbon-based superlattices and demonstrate that BZ oscillations lead to clear-cut features in the $I-V$ characteristics. To this end, we model a graphene nanoribbon of width $W$ and length $L$ by the tight-binding Hamiltonian of Eq. (1), now with a periodic electrostatic potential $V_{0} \sin (2 \pi x / a)$ leading to a superlattice miniband structure, as shown in the inset of Fig. 6(a). A small constant mass term $M(x)=M_{0}$ is additionally considered, which opens up a gap commonly present in experiments on graphene nanoribbons.$^{41}$ We assume a linear potential drop $\mathrm{eV}_{\mathrm{SD}} x / L$ due to the source-drain voltage $V_{\mathrm{SD}}$ between the graphene leads at $x= \pm L / 2$. The current is calculated by means of the Landauer-Büttiker formalism, ${ }^{42}$

$$
I\left(V_{\mathrm{SD}}\right)=\frac{2 e}{h} \int_{-\infty}^{\infty} T\left(E, V_{\mathrm{SD}}\right)\left[f^{+}(E)-f^{-}(E)\right] d E,
$$

with the Fermi functions $f^{ \pm}(E)=\{1+\exp [(E \mp$ $\left.\left.\left.V_{\mathrm{SD}} / 2\right) / k_{\mathrm{B}} T\right]\right\}^{-1}$.
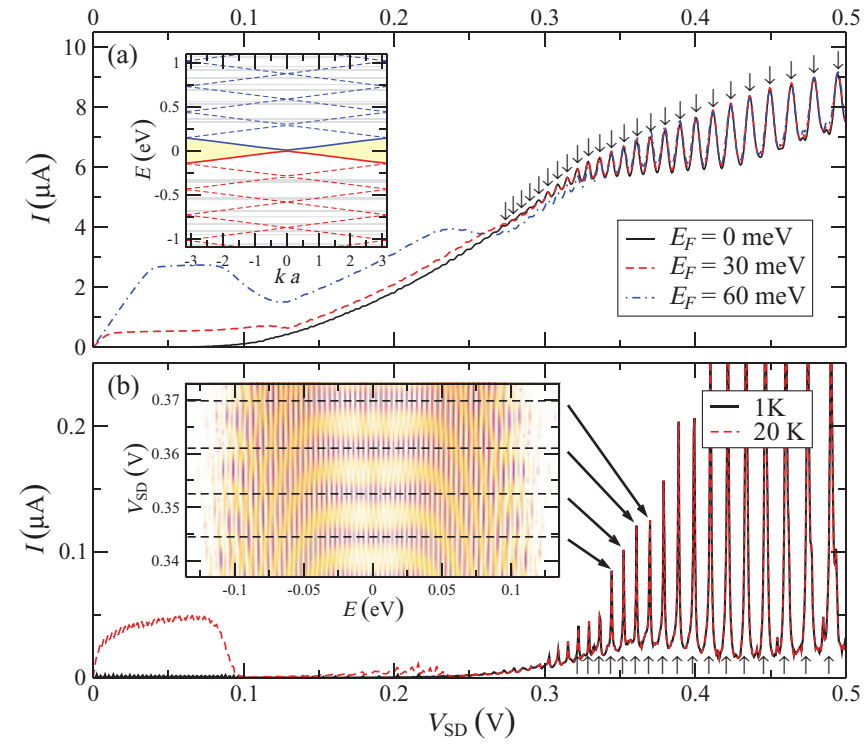

FIG. 6. (Color online) Current-voltage characteristics for graphene nanoribbon superlattices $\left(L=3000 \sqrt{3} a_{0}, W=10 a_{0}, a=\right.$ $\left.30 \sqrt{3} a_{0}, V_{0}=500 \mathrm{meV}\right)$ for (a) different Fermi energies $\left(M_{0}=\right.$ $20 \mathrm{meV}, T=20 \mathrm{~K})$ and (b) different temperatures $\left(M_{0}=50 \mathrm{meV}\right.$, $\left.E_{F}=0\right)$ showing pronounced signatures of Bloch-Zener oscillations at higher bias. Arrows mark expected peak positions from phase condition (17). Upper inset: Band structure (for $M_{0}=20 \mathrm{meV}$ ). Lower inset: Transmission map $T\left(E, V_{\mathrm{SD}}\right)$ used in Eq. (18) to get the current of panel (b); dark colors represent high transmissions.

As shown in Fig. 6, the current through the nanoribbon is governed by a conventional increase with the bias window for small $V_{\mathrm{SD}}$, followed by a region of negative differential conductance typical for superlattices. At higher bias, $V_{\mathrm{SD}}>0.3 \mathrm{~V}$, we observe the emergence of distinct current oscillations that get more pronounced with increasing gap size; see Fig 6(b). Due to the bias between the source and drain electrode, the particles traversing the superlattice must change their electron-hole character. However, states performing $\mathrm{BZ}$ oscillations exhibit transitions between the two carrier types only for certain $V_{\mathrm{SD}}=e E_{D} L$ when the phase $\phi$ fulfills the condition of Eq. (17), as shown in the previous section. Consequently, the current is strongly enhanced if this is fulfilled. As shown in Figs. 6(a) and 6(b), the current peaks calculated by Eq. (18) perfectly coincide with the expected voltages (marked by vertical arrows) deduced by extracting the area $\mathcal{A}$ in momentum space from the minibands around the Fermi energy shown as the shaded area in the inset of Fig. 6(a). Vice versa, the experimental observation of BZ peaks in the $I-V$ characteristics would allow for "measuring" the miniband structure.

A closer look at the transmission values $T\left(E, V_{\mathrm{SD}}\right)$ [see inset Fig. 6(b)] reveals a rhombic structure, which features pronounced transmission maxima piled up at these particular values of $V_{\mathrm{SD}}$ (dashed lines). Since these maxima are present for various energies in the conductance window, the resulting current is fairly independent of the exact Fermi energy [see Fig. 6(a)] and temperature [see Fig. 6(b)]. 


\section{BZ OSCILLATIONS AND TRANSPORT IN HGTE-BASED SUPERLATTICES}

A different setup featuring BZ oscillations can be created from a strip etched out of the two-dimensional topological insulator based on mercury telluride (HgTe). ${ }^{27,28}$ We describe the electronic properties of the underlying $\mathrm{HgTe}$ heterostructure by the Hamiltonian ${ }^{25}$

$$
H=\left(\begin{array}{cccc}
C_{k}+M_{k} & A k_{+} & 0 & 0 \\
A k_{-} & C_{k}-M_{k} & 0 & 0 \\
0 & 0 & C_{k}+M_{k} & -A k_{-} \\
0 & 0 & -A k_{+} & C_{k}-M_{k}
\end{array}\right)
$$

where $k_{ \pm}=k_{x} \pm \mathrm{i} k_{y}, \boldsymbol{k}^{2}=k_{x}^{2}+k_{y}^{2}, C_{k}=-D \boldsymbol{k}^{2}$, and $M_{k}=$ $M-B \boldsymbol{k}^{2}$. We assume an $\mathrm{HgTe} / \mathrm{HgCdTe}$ heterostructure with a quantum well width of $7.0 \mathrm{~nm}$ featuring topological edge states and leading to material parameters $A, B, D$, and $M$, as typically used in the literature. ${ }^{43}$ As for the graphene nanoribbon, we can create two different types of superlattices with a masslike modulation and an electrostatic modulation. For a HgTe strip, the mass modulation can be achieved by modulating the width of the ribbon, for example, by

$$
W(x)=\frac{W_{0}+W_{1}}{2}-\frac{W_{0}-W_{1}}{2} \sin \left(\frac{2 \pi x}{a}\right),
$$

where $W_{0}$ and $W_{1}$ are the maximum and minimum width, and $a$ is the periodicity. The finite width of the $\mathrm{HgTe}$ nanoribbon allows for a hybridization of the edge states with the same spin at the opposite boundaries, leading to a small gap in the band structure. ${ }^{44,45}$ Accordingly, the modulation of the width corresponds to a modulation of the mass gap. The resulting miniband structure from the two-dimensional system, shown in Fig. 7(a), is obtained numerically by Lanczos diagonalization and exhibits various Landau-Zener anticrossings within the bulk band gap of HgTe, which suggest BZ oscillations.

In order to study the electron dynamics, we calculate the COM motion of Gaussian-shaped edge-state wave packets. Initially, the wave packet is localized on one edge and the direction of motion is determined by its spin. The array of multiple constrictions enables tunneling between the edges. As a consequence, an inversion of the direction of motion is possible, leading to Bloch and BZ oscillations. As shown in Fig. 7(b), the resulting frequency spectrum features the expected rhombic pattern in between the frequencies of the conventional Bloch oscillations (white dashed lines). Compared to the graphene system [see Fig. 3(e)], we observe more complicated, superimposed structures because of the whole sequence of multiple anticrossings in the band structure that affect BZ oscillations.

As for graphene, we further study the transport properties of HgTe strips of constant width and a periodically modulated electrostatic potential resulting in a supercell band structure, shown in the inset of Fig. 7(c). The small gap between the electron and the hole states is attributed to the finite ribbon width of $150 \mathrm{~nm}$. We choose the Fermi energy close to the band crossing of the topological edge states and calculate the current using Eq. (18). Besides a strong negative-differential conductance at lower bias, we get the signatures of BZ

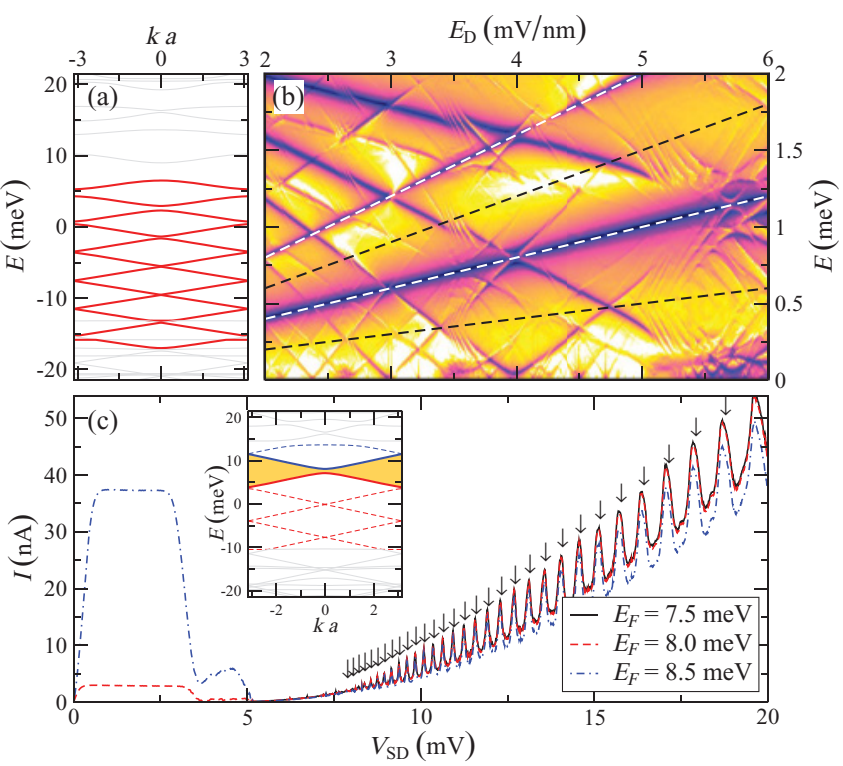

FIG. 7. (Color online) Bloch and Bloch-Zener oscillations in spatially modulated two-dimensional HgTe nanoribbons. (a) Band structure for a $\mathrm{HgTe}$ nanoribbon with periodically modulated width $W(x)$ [Eq. (20)] ranging from $W_{0}=300$ to $W_{1}=50 \mathrm{~nm}$ and periodicity $a=200 \mathrm{~nm}$. (b) Frequency spectrum $E=\hbar \omega$ of the wave-packet center-of-mass motion as a function of drift field $E_{D}$. Dashed lines indicate the frequencies of the Bloch oscillations. (c) $I$ $V_{\mathrm{SD}}$ characteristics of a nanoribbon with constant width $W=150 \mathrm{~nm}$ and electrostatic modulation $V(x)=V_{0} \sin (2 \pi x / a)$. Small vertical arrows mark the expected maxima from phase condition (17). Inset: Corresponding miniband structure.

oscillations for $V_{\mathrm{SD}}>9 \mathrm{mV}$, as shown in Fig. 7(c). Similar to the calculations for the graphene superlattice, the oscillations are independent of the exact choice of the Fermi level. The peak positions are in good accordance with the expected series of drift voltages marked by arrows in Fig. 7(c) obtained from Eq. (17), where $\mathcal{A}$ is extracted from the bands around the Fermi energy shown as the shaded area in the inset.

\section{CONCLUSION}

In this paper, we showed that Bloch-Zener oscillations appear naturally in superlattices made of materials with a Dirac-like spectrum, highlighting interference between the electron and hole states. The characteristics of these oscillations are explained by a one-dimensional model Hamiltonian and numerically confirmed for realistic setups by means of wave-packet simulations for graphene and topological insulator ribbons. Furthermore, we demonstrated that Bloch-Zener oscillations manifest themselves as a regular sequence of pronounced current peaks in quantum transport, besides the wellknown negative differential conductance at low bias, which is a signature of conventional Bloch oscillations. The sequence of current peaks associated with the Bloch-Zener oscillations is intimately linked to the underlying miniband structure.

We suggest transport measurements through graphene nanoribbons and $\mathrm{HgTe}$ strips as promising experimental setups that feature Bloch-Zener oscillations. For single layer graphene and topological insulators, the periodic electrostatic 
potential can be imprinted by an array of top gates. The gap between the electron and hole states can be tuned by the width of the considered nanoribbons. In the case of bilayer graphene, the gap can also be created via a potential difference in the $z$ direction induced by top gating. The calculations presented here have been performed for clean, disorder-free, and coherent systems. However, preliminary numerical calculations for graphene-based superlattices with disorder indicate that Bloch-Zener oscillations are still visible if the mean free path exceeds several periods of the superlattice. This is promising with respect to their experimental detection in solid-state-based samples.

We finally note that signatures of the Bloch-Zener oscillations presented have been recently observed with ultracold, fermionic $\mathrm{K}$ atoms due to the Dirac points with small mass gaps emerging in tunable optical honeycomb lattices. ${ }^{46}$

Note added in proof. Recently, we became aware of Ref. 47 where the Bloch-Zener oscillations of collective excitations in narrow zigzag-shaped optical lattices are studied theoretically.

\section{ACKNOWLEDGMENTS}

This work is supported by Deutsche Forschungsgemeinschaft (GRK 1570 and joined DFG-JST Forschergruppe Topological Electronics). We thank T. Hartmann, F. Tkatschenko, and D. Ryndyk for useful conversations.
${ }^{1}$ F. Bloch, Z. Phys. A 52, 555 (1929).

${ }^{2}$ C. Zener, Proc. R. Soc. London A 145, 523 (1934).

${ }^{3}$ J. Feldmann et al., Phys. Rev. B 46, 7252 (1992).

${ }^{4}$ K. Leo et al., Solid State Commun. 84, 943 (1992).

${ }^{5}$ C. Waschke et al., Phys. Rev. Lett. 70, 3319 (1993).

${ }^{6}$ M. Ben Dahan et al., Phys. Rev. Lett. 76, 4508 (1996).

${ }^{7}$ S. Wilkinson et al., Phys. Rev. Lett. 76, 4512 (1996).

${ }^{8}$ T. Pertsch et al., Phys. Rev. Lett. 83, 4752 (1999).

${ }^{9}$ R. Morandotti et al., Phys. Rev. Lett. 83, 4756 (1999).

${ }^{10}$ H. Sanchis-Alepuz, Y. Kosevich, and J. Sánchez-Dehesa, Phys. Rev. Lett. 98, 134301 (2007).

${ }^{11}$ H. Fukuyama, R. Bari, and H. Fogedby, Phys. Rev. B 8, 5579 (1973).

${ }^{12}$ J. Rotvig, A.-P. Jauho, and H. Smith, Phys. Rev. Lett. 74, 1831 (1995).

${ }^{13}$ D. Hone and X.-G. Zhao, Phys. Rev. B 53, 4834 (1996).

${ }^{14}$ B. Breid, D. Witthaut, and H. Korsch, New J. Phys. 8, 110 (2006).

${ }^{15}$ B. Breid, D. Witthaut, and H. Korsch, New J. Phys. 9, 62 (2007).

${ }^{16}$ P. Abumov and D. W. L. Sprung, Phys. Rev. B 75, 165421 (2007).

${ }^{17}$ Y. Shimada, N. Sekine, and K. Hirakawa, Appl. Phys. Lett. 84, 4926 (2004).

${ }^{18}$ F. Dreisow et al., Phys. Rev. Lett. 102, 076802 (2009).

${ }^{19}$ S. Kling, T. Salger, C. Grossert, and M. Weitz, Phys. Rev. Lett. 105, 215301 (2010).

${ }^{20} \mathrm{P}$. Wallace, Phys. Rev. 71, 622 (1947).

${ }^{21}$ K. Novoselov et al., Science 306, 666 (2004).

${ }^{22}$ Y. Zhang, Y.-W. Tan, H. Stormer, and P. Kim, Nature (London) 438, 201 (2005).

${ }^{23}$ C. Kane and E. Mele, Phys. Rev. Lett. 95, 226801 (2005).

${ }^{24}$ C. Kane and E. Mele, Phys. Rev. Lett. 95, 146802 (2005).

${ }^{25}$ B. Bernevig, T. Hughes, and S.-C. Zhang, Science 314, 1757 (2006).
${ }^{26}$ B. Bernevig and S.-C. Zhang, Phys. Rev. Lett. 96, 106802 (2006).

${ }^{27}$ M. König et al., Science 318, 766 (2007).

${ }^{28}$ A. Roth et al., Science 325, 294 (2009).

${ }^{29}$ C.-H. Park et al., Nature Phys. 4, 213 (2008).

${ }^{30}$ M. Barbier, P. Vasilopoulos, and F. Peeters, Phys. Rev. B 81, 075438 (2010).

${ }^{31}$ L. Brey and H. Fertig, Phys. Rev. Lett. 103, 046809 (2009).

${ }^{32}$ G. J. Ferreira, M. N. Leuenberger, D. Loss, and J. C. Egues, Phys. Rev. B 84, 125453 (2011).

${ }^{33}$ J. Meyer, C. Girit, M. Crommie, and A. Zettl, Appl. Phys. Lett. 92, 123110 (2008).

${ }^{34}$ D. Dragoman and M. Dragoman, Appl. Phys. Lett. 93, 103105 (2008).

${ }^{35}$ K. Nakada, M. Fujita, G. Dresselhaus, and M. Dresselhaus, Phys. Rev. B 54, 17954 (1996).

${ }^{36}$ V. Krueckl and T. Kramer, New J. Phys. 11, 093010 (2009).

${ }^{37}$ See Supplemental Material at http://link.aps.org/supplemental/ 10.1103/PhysRevB.85.115433 at for a video of Bloch-Zener oscillations on a graphene superlattice.

${ }^{38}$ L. D. Landau, Phys. Z. Sowjetunion 2, 46 (1932).

${ }^{39}$ C. Zener, Proc. R. Soc. London A 137, 696 (1932).

${ }^{40}$ E. C. G. Stueckelberg, Helv. Phys. Acta 5, 369 (1932).

${ }^{41}$ M. Han, J. Brant, and P. Kim, Phys. Rev. Lett. 104, 056801 (2010).

${ }^{42}$ M. Büttiker, Y. Imry, R. Landauer, and S. Pinhas, Phys. Rev. B 31, 6207 (1985).

${ }^{43}$ M. König et al., J. Phys. Soc. Jpn. 77, 031007 (2008).

${ }^{44}$ B. Zhou, H. Z. Lu, R. L. Chu, S. Q. Shen, and Q. Niu, Phys. Rev. Lett. 101, 246807 (2008).

${ }^{45}$ V. Krueckl and K. Richter, Phys. Rev. Lett. 107, 086803 (2011).

${ }^{46}$ L. Tarruell, D. Greif, T. Uehlinger, G. Jotzu, and T. Esslinger, Nature 483, 302 (2012).

${ }^{47}$ E. Arévalo and L. Morales-Molina, EPL 96, 60011 (2011). 